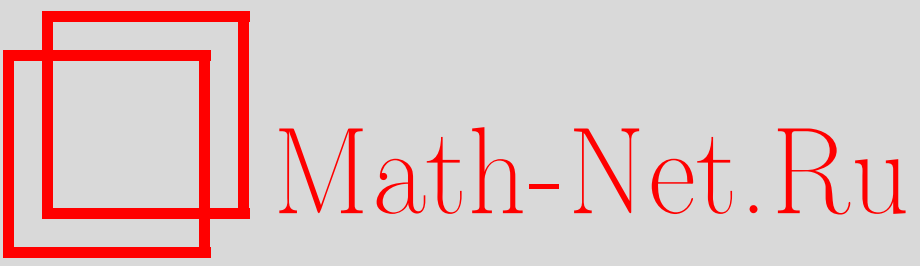

М. Горжини, Д. П. Санкович, Н. И. Танака, Об одной модели неидеального бозе-газа, ТМФ, 1999, том 120, номер 1, 130-143

DOI: https://doi.org/10.4213/tmf765

Использование Общероссийского математического портала Math-Net.Ru подразумевает, что вы прочитали и согласны с пользовательским соглашением

http://www.mathnet.ru/rus/agreement

Параметры загрузки:

IP: 3.80 .181 .102

26 апреля 2023 г., 06:47:22 


\author{
ТЕОРЕТИЧЕСКАЯ \\ И МАТЕМАТИЧЕСКАЯ \\ ФИЗИКА \\ Том 120, № 1 \\ июль, 1999
}

(С) 1999 г. $\quad$ М. Горжини* , Д. П. Санкович ${ }^{\dagger}$, Н. И. Танака

\title{
ОБ ОДНОЙ МОДЕЛИ НЕИДЕАЛЬНОГО БОЗЕ-ГАЗА
}

Рассмотрено полиномиальное обобщение модели Хуанга-Дэвиса в теории неидеального бозе-газа. Доказана выполнимость условия гауссовой доминантности для всех значений химического потенциала. Показано, что нижняя граница критической температуры модели Хуанга-Дэвиса, полученная методом инфракрасных оценок, совпадает с точным значением этой величины в теории Дэвиса. С помощью метода больших уклонений доказана возможность обобщенной бозе-конденсации в полиномиальной модели.

\section{1. ВВЕДЕНИЕ}

При изучении системы взаимодействующих бозонов в приближении среднего поля энергия взаимодействия описывается слагаемым $a \widehat{N}^{2} /(2 V)$, добавляемым к свободному гамильтониану. Положительньй параметр $а$ задает интенсивность взаимодействия, $\widehat{N}$ - оператор числа частиц, $V$ - объем ограниченной измеримой области $\nu$-мерного пространства $R^{\nu}$ (или решетки $Z^{\nu}$ ), в которой расположены бозоны. Смысл слагаемого $a \widehat{N}^{2} /(2 V)$ может быть понят в рамках классической оптики, когда движение каждой частицы в системе рассматривается как распространение света в однородной оптической среде (так называемая аппроксимация с помощью показателя преломления). При этом добавка к энергии пропорциональна плотности. Такая модель взаимодействующих бозонов обычно называется моделью Хуанга-Дэвиса $[1,2]$.

В работах $[3,4]$ было показано, как данная модель описывает парное отталкивание бозонов в пределе слабого дальнодействия. При малых плотностях модель Хуанга-Дэвиса иногда рассматривается в качестве приближения для парного взаимодействия с двухчастичным потенциалом $U(x-y)=a \delta(x-y)$.

В статье [2] с помощью вероятностных методов, включающих, в частности, центральную предельную теорему, было проведено строгое изучение обобшенной модели Хуанга-Дэвиса в бесконечном объеме. В данной модели взаимодействие имеет вид $V f(\hat{n})$, где $\hat{n}=\widehat{N} / V$. Функция $f$ предполагается непрерывно дифференцируемой на полуоси $[0, \infty)$ и удовлетворяющей условиям $f(0)=0$ и $\lim _{x \rightarrow \infty} f^{\prime}(x)=+\infty$. Было

\footnotetext{
* Университет Ла-Серены, Ла-Серена, Чили

${ }^{\dagger}$ Математический институт им.В.А.Стеклова РАН, Москва, Россия

‡Университет Сан-Паулу, Сан-Паулу, Бразилия
} 
получено точное выражение для средней плотности числа частиц как функции химического потенциала и показано наличие сингулярности у функции плотности в точке бозе-эйнштейновской конденсации при определенных условиях на вид функции $f$. Было также доказано, что, в отличие от идеального бозонного газа, неидеальньй бозе-газ Хуанга-Дэвиса имеет (в бесконечном объеме) одни и те же свойства как в большом каноническом ансамбле, так и в каноническом ансамбле. Рассматриваемая модель оказалась устойчивой при малых возмушениях. В этой модели имеет место обычная бозе-эйнштейновская конденсация.

В работах $[5,6]$ детально изучалась проблема конденсации Бозе-Эйнштейна в газе невзаимодействуюших бозонов. В этих работах установлено различие между макроскопическим заполнением основного состояния (обычная бозе-эйнштейновская конденсация) и так называемой обобщенной конденсацией. Макроскопическое заполнение основного состояния происходит тогда, когда число частищ в этом состоянии становится пропорциональным объему. Обобщенная же конденсация возникает, когда число частиц с энергиями, лежашими в малой окрестности нуля, становится пропорциональным объему. Величина макроскопического заполнения сильно зависит от формы сосуда, в котором расположен бозонный газ. Наличие обобшенной конденсации связано только с условием, что плотность $\rho$ принимает критическое значение $\rho_{\mathrm{c}}$. Обобшенная конденсация оказалась устойчивой при возмушении свободного гамильтониана средним полем.

Изучение некоторых модельных систем неидеального бозе-газа с помощью метода больших уклонений $[7]$ проведено в работах $[8-11]$. В статьях $[12,13]$ для исследования подобных моделей применялся метод инфракрасных оценок и было доказано сушествование при конечной температуре макроскопического заполнения основного состояния.

\section{2. ОБОБЩЕННАЯ МОДЕЛЬ ХУАНГА-ДЭВИСА}

Пусть $\Lambda \subset R^{\nu}$ - открытая область с гладкой границей $\partial \Lambda$ и единичным объемом и пусть $L \geqslant 1$. Определим область $\Lambda_{L}=\{L x \mid x \in \Lambda\}$ и пространство комплексных квадратично-интегрируемых функций на ней $\mathcal{H}^{L}=L^{2}\left(\Lambda_{L}\right)$. Объем области $\Lambda_{L}$ обозначим как $\left|\Lambda_{L}\right| \equiv V_{L}$. Оператор $\widehat{S}^{L}=-\triangle$ самосопряжен на пространстве $\mathcal{H}^{L}$, имеет дискретный спектр

$$
0=E_{L}(0)<E_{L}(1) \leqslant E_{L}(2) \leqslant \cdots
$$

и собственные функции $\left\{\phi_{k}(x)\right\}$, удовлетворяюшие одночастичной задаче

$$
-\frac{1}{2} \triangle \phi_{k}(x)=E_{L}(k) \phi_{k}(x)
$$

с некоторыми заданными на $\partial \Lambda$ граничными условиями. Набор функций $\left\{\phi_{k}(x)\right\}$ образует базис в пространстве $L^{2}\left(\Lambda_{L}\right)$.

Любая функция $\psi\left(x_{1}, \ldots, x_{N}\right) \in L^{2}\left(\Lambda_{L}^{N}\right)$ может быть разложена по базису

$$
\left\{\prod_{j=1}^{N} \phi_{k_{j}}\left(x_{j}\right)\right\} .
$$


Функции

$$
\psi_{k}\left(x_{1}, \ldots, x_{N}\right)=\bigotimes_{j=1}^{N} \phi_{k_{j}}\left(x_{j}\right)
$$

где $k=\left\{k_{1}, k_{2}, \ldots, k_{N}\right\}$, образуют в гильбертовом пространстве

$$
\mathcal{H}^{N, L}=\bigotimes_{i=1}^{N} \mathcal{H}^{L}
$$

полный ортонормированный базис со скалярным произведением

$$
\left(\psi_{k}, \psi_{l}\right)=\prod_{j=1}^{N}\left(\phi_{k_{j}}, \phi_{l_{j}}\right)
$$

Будем рассматривать подпространство $\mathcal{H}_{+}^{N, L}$, являюшееся сужением пространства $\mathcal{H}^{N, L}$ до симметричных относительно перестановки аргументов функций (бозе-статистика). Функции из подпространства $\mathcal{H}_{+}^{N, L}$ однозначно разлагаются по базису симметризованных функций $\left\{\psi_{k}^{+}\right\}$, построенному на основе базиса $\left\{\psi_{k}\right\}$ с помощью симметризации

$$
\left(S_{+}^{N} \psi_{k}\right)\left(x_{1}, \ldots, x_{N}\right)=\frac{1}{N !} \sum_{p} P \psi_{k}\left(x_{1}, \ldots, x_{N}\right)=\psi_{k}^{+}\left(x_{1}, \ldots, x_{N}\right)
$$

где $S_{+}^{N}$ - оператор симметризации и $P$ - оператор перестановки аргументов, $P \psi\left(x_{1}, \ldots\right.$ $\left.\ldots, x_{N}\right)=\psi\left(x_{p_{1}}, \ldots, x_{p_{N}}\right)$. Оператор симметризации является ортогональным проектором пространства $\mathcal{H}^{N, L}$ на пространство $\mathcal{H}_{+}^{N, L}$. Набор функций $\left\{\psi_{k}^{+}\left(x_{1}, \ldots, x_{N}\right)\right\}$ образует ортогональный базис в пространстве $\mathcal{H}_{+}^{N, L}$.

Положим $\mathcal{H}_{+}^{0, L}=C$. Тогда симметричное пространство Фока $\mathcal{F}_{+}\left(\mathcal{H}^{L}\right)$ является прямой ортогональной суммой

$$
\mathcal{F}_{+}\left(\mathcal{H}^{L}\right)=\bigoplus_{N=0}^{\infty} \mathcal{H}_{+}^{N, L}
$$

Определим модельный гамильтониан неидеального бозе-газа соотношением

$$
\widehat{H}^{L}=\widehat{H}_{0}+\frac{a}{r V_{L}^{r-1}} \widehat{N}^{r}
$$

Оператор $\widehat{H}_{0}$ действует в пространстве $\mathcal{F}_{+}\left(\mathcal{H}^{L}\right)$ и отвечает свободному гамильтониану, построенному соответствуюшим образом [14] из оператора $\widehat{S}^{L}$. Оператор $\widehat{N}$ задает оператор числа частиц,

$$
\widehat{N} \psi_{k}^{+}\left(x_{1}, \ldots, x_{N}\right)=N \psi_{k}^{+}\left(x_{1}, \ldots, x_{N}\right) .
$$

В формуле (1) целое число $r \geqslant 2$. В дальнейшем будем использовать бозе-операторы рождения и уничтожения $[14,15]$, удовлетворяюшие каноническим коммутационным соотношениям

$$
\left[\hat{a}_{i}, \hat{a}_{j}^{\dagger}\right] \equiv \hat{a}_{i} \hat{a}_{j}^{\dagger}-\hat{a}_{j}^{\dagger} \hat{a}_{i}=\delta_{i, j}
$$


Модель бозе-газа с гамильтонианом (1) назовем полиномиальным обобщением модели Хуанга-Дэвиса. Используя описанную в разделе 1 оптическую аналогию, можно сказать, что данная полиномиальная модель соответствует учету дисперсии показателя преломления.

Зададим состояние термодинамического равновесия соотношением

$$
\langle\ldots\rangle_{\widehat{H}^{L}}=\frac{\operatorname{Tr} \ldots e^{-\beta\left(\hat{H}^{L}-\mu \widehat{N}\right)}}{\operatorname{Tr} e^{-\beta\left(\hat{H}^{L}-\mu \widehat{N}\right)}} .
$$

Средняя плотность в конечном объеме $V_{L}$ есть $\rho_{V_{L}}=\langle\hat{n}\rangle_{\hat{H}^{L}}$. При переходе к термодинамическому пределу

$$
\rho=\lim _{L \rightarrow \infty} \rho_{V_{L}} .
$$

Оператор $\hat{n}$ называется оператором плотности, $\mu$ и $\beta$ представляют собой химический потенциал и обратную температуру, соответственно.

Определим в фоковском пространстве множество финитных векторов

$$
\mathcal{M}=\left\{\phi=\left\{\phi^{(N)}\right\}_{N=0}^{\infty} \in \mathcal{F}_{+}\left(\mathcal{H}^{L}\right) \mid \exists N_{\phi}: \phi^{(N)}=0 \text { для всех } N>N_{\phi}\right\} .
$$

Очевидно, что замыкание $\overline{\mathcal{M}}=\mathcal{F}_{+}\left(\mathcal{H}^{L}\right)$. Операторы $\widehat{H}_{0}$ и $\widehat{N}$ не ограничены на $\mathcal{M}$.

\section{3. ГАУССОВА ДОМИНАНТНОСТЬ}

Большая статистическая сумма системы с гамильтонианом (1) задается соотношениeм

$$
\begin{aligned}
Z_{\beta, \mu}\left[\widehat{H}^{L}\right] & =\operatorname{Tr}\left[e^{-\beta\left(\hat{H}^{L}-\mu \widehat{N}\right)}\right]=\sum_{N=0}^{\infty} \operatorname{Tr}_{\mathcal{H}_{+}^{N, L}}\left[e^{-\beta\left(\hat{H}^{L}-\mu \widehat{N}\right)}\right]= \\
& =\sum_{N=0}^{\infty} e^{\beta \mu N} \operatorname{Tr}_{\mathcal{H}_{+}^{N, L}}\left[e^{-\beta \hat{H}^{N, L}}\right]
\end{aligned}
$$

где $\widehat{H}^{N, L}=\left.\widehat{H}^{L}\right|_{\mathcal{H}_{+}^{N, L}}, Z_{\beta, \mu}\left[\widehat{H}^{L}\right]_{N=0}=1,\left[\widehat{H}^{L}\right]_{N=1}=\widehat{H}_{0}$ и след $\operatorname{Tr}_{\mathcal{H}_{+}^{N, L}}(\cdot)$ обозначает ограничение следа на пространство $\mathcal{H}_{+}^{N, L}$.

Рассмотрим семейство операторов

$$
\begin{aligned}
\widehat{H}^{L}\left(\left\{\frac{h_{j}}{\sqrt{V_{L}}}\right\}\right) & =\sum_{j \geqslant 1} \lambda(j)\left(\hat{a}_{j}^{\dagger}-\frac{h_{j}^{*}}{\sqrt{V_{L}}}\right)\left(\hat{a}_{j}-\frac{h_{j}}{\sqrt{V_{L}}}\right)+\frac{a}{r V_{L}^{r-1}} \widehat{N}^{r} \equiv \\
& \equiv \widehat{H}_{0}\left(\left\{\frac{h_{j}}{\sqrt{V_{L}}}\right\}\right)+\frac{a}{r V_{L}^{r-1}} \widehat{N}^{r},
\end{aligned}
$$

где $\lambda(j)=E_{L}(j)$ и только конечное число параметров $h_{j} \in C$ отлично от нуля. Для каждого $N$ существует такой унитарный оператор $\widehat{U}_{N}$, что

$$
\operatorname{Tr}_{\mathcal{H}_{+}^{N, L}}\left[\exp \left\{-\beta \widehat{H}_{0}\left(\left\{\frac{h_{j}}{\sqrt{V_{L}}}\right\}\right)\right\}\right]=\operatorname{Tr}_{\mathcal{H}_{+}^{N, L}}\left[\widehat{U}_{N}^{+} e^{-\beta \widehat{H}_{0}} \widehat{U}_{N}\right]=\operatorname{Tr}_{\mathcal{H}_{+}^{N, L}}\left[e^{-\beta \widehat{H}_{0}}\right] .
$$


Можно записать, что

где

$$
\begin{aligned}
& \operatorname{Tr}\left[\exp \left\{-\beta\left(\widehat{H}^{L}\left(\left\{\frac{h_{j}}{\sqrt{V_{L}}}\right\}\right)-\mu \widehat{N}\right)\right\}\right]= \\
& \quad=\exp \left(\frac{\beta \mu^{2} r V_{L}^{r-1}}{4 a}\right) \operatorname{Tr}\left[\exp \left\{-\beta\left(\widehat{H}_{0}\left(\left\{\frac{h_{j}}{\sqrt{V_{L}}}\right\}\right)+\frac{a}{r V_{L}^{r-1}} \widehat{W}\right)\right\}\right]
\end{aligned}
$$

$$
\widehat{W}=\left(\frac{\mu r V_{L}^{r-1}}{2 a}-\widehat{N}\right)^{2}+\widehat{N}^{r}-\widehat{N}^{2}
$$

Ясно, что оператор $\left.\widehat{W}\right|_{\mathcal{H}_{+}^{N, L}}$ положителен и самосопряжен при $r \geqslant 2$.

Воспользуемся теперь неравенством Голдена-Томпсона [16], справедливым для самосопряженных операторов $\hat{A}$ и $\widehat{B}$,

$$
\operatorname{Tr}\left(e^{\hat{A}+\widehat{B}}\right) \leqslant \operatorname{Tr}\left(e^{\hat{A}} e^{\widehat{B}}\right) .
$$

Применяя его в нашем случае, для любых $\mu$ имеем

$$
\begin{aligned}
& \operatorname{Tr}\left[\exp \left\{-\beta\left(\widehat{H}^{L}\left(\left\{\frac{h_{j}}{\sqrt{V_{L}}}\right\}\right)-\mu \widehat{N}\right)\right\}\right] \\
& \quad \leqslant \exp \left(\frac{\beta \mu^{2} r V_{L}^{r-1}}{4 a}\right) \operatorname{Tr}\left[\exp \left\{-\beta \widehat{H}_{0}\left(\left\{\frac{h_{j}}{\sqrt{V_{L}}}\right\}\right)\right\} \exp \left\{-\frac{\beta a}{r V_{L}^{r-1}} \widehat{W}\right\}\right] .
\end{aligned}
$$

Вспомнив определение следа, находим

$$
\begin{aligned}
\operatorname{Tr}_{\mathcal{H}_{+}^{N, L}} & {\left[\exp \left\{-\beta \widehat{H}_{0}\left(\left\{\frac{h_{j}}{\sqrt{V_{L}}}\right\}\right)\right\} \exp \left\{-\frac{\beta a}{r V_{L}^{r-1}} \widehat{W}\right\}\right]=} \\
= & \sum_{k=\left\{k_{1}, \ldots, k_{N}\right\}}\left(\psi_{k}^{+}\left(x_{1}, \ldots, x_{N}\right),\left[\exp \left\{-\beta \widehat{H}_{0}\left(\left\{\frac{h_{j}}{\sqrt{V_{L}}}\right\}\right)\right\} \times\right.\right. \\
\times & \left.\left.\exp \left\{-\frac{\beta a}{r V_{L}^{r-1}} \widehat{W}\right\}\right] \psi_{k}^{+}\left(x_{1}, \ldots, x_{N}\right)\right) .
\end{aligned}
$$

Заметив теперь, что

$$
\begin{aligned}
& \exp \left(-\beta \frac{a}{r V_{L}^{r-1}} \widehat{W}\right) \psi_{k}^{+}\left(x_{1}, \ldots, x_{N}\right)= \\
& \quad=\exp \left\{-\beta \frac{a}{r V_{L}^{r-1}}\left[\left(\frac{\mu r V_{L}^{r-1}}{2 a}-N\right)^{2}+N^{r}-N^{2}\right]\right\} \psi_{k}^{+}\left(x_{1}, \ldots, x_{N}\right),
\end{aligned}
$$

перепишем неравенство (3) в виде

$$
\begin{aligned}
\operatorname{Tr}[\exp & \left.\left\{-\beta\left(\widehat{H}^{L}\left(\left\{\frac{h_{j}}{\sqrt{V_{L}}}\right\}\right)-\mu \widehat{N}\right)\right\}\right] \leqslant \exp \left(\frac{\beta \mu^{2} r V_{L}^{r-1}}{4 a}\right) \times \\
\times & \sum_{N=0}^{\infty} \exp \left\{-\frac{\beta a}{r V_{L}^{r-1}}\left[\left(\frac{\mu r V_{L}^{r-1}}{2 a}-N\right)^{2}+N^{r}-N^{2}\right]\right\} \times \\
\times & \operatorname{Tr}_{\mathcal{H}_{+}^{N, L}}\left[\exp \left\{-\beta \widehat{H}_{0}\left(\left\{\frac{h_{j}}{\sqrt{V_{L}}}\right\}\right)\right\}\right] .
\end{aligned}
$$


Учитывая равенства $(2)$ и факт коммутативности операторов $\widehat{N}$ и $\widehat{H}_{0}$ между собой, находим из неравенства (4), что

$$
\begin{aligned}
\operatorname{Tr}[\exp \{ & \left.\left.-\beta\left(\widehat{H}^{L}\left(\left\{\frac{h_{j}}{\sqrt{V_{L}}}\right\}\right)-\mu \widehat{N}\right)\right\}\right] \leqslant \exp \left(\frac{\beta \mu^{2} r V_{L}^{r-1}}{4 a}\right) \times \\
& \times \sum_{N=0}^{\infty} \exp \left\{-\frac{\beta a}{r V_{L}^{r-1}}\left[\left(\frac{\mu r V_{L}^{r-1}}{2 a}-N\right)^{2}+N^{r}-N^{2}\right]\right\} \operatorname{Tr}_{\mathcal{H}_{+}^{N, L}}\left[e^{-\beta \hat{H}_{0}}\right]= \\
= & \operatorname{Tr}\left[e^{-\beta\left(\hat{H}^{L}-\mu \widehat{N}\right)}\right] .
\end{aligned}
$$

Полученный результат можно сформулировать в виде следуюшей теоремы.

Теорема 1. Для полиномиального обобщения модели Хуанга-Дәвиса (1) при любых $\beta \geqslant 0$ и н имеет место условие гауссовой доминантности

$$
Z_{\beta, \mu}\left[\widehat{H}^{L}\left(\left\{\frac{h_{j}}{\sqrt{V_{L}}}\right\}\right)\right] \leqslant Z_{\beta, \mu}\left[\widehat{H}^{L}\right] .
$$

В большом каноническом ансамбле термодинамический потенциал (давление) для системы с гамильтонианом $\widehat{S}$ определяется соотношением

$$
P_{\beta, \mu}[\widehat{S}]=\frac{1}{\beta V_{L}} \ln \operatorname{Tr} e^{-\beta(\widehat{S}-\mu \widehat{N})} .
$$

Рассмотрим оператор $\widehat{S}_{\lambda}=\widehat{S}+\lambda \widehat{U}$, где $\lambda \in R^{1}$. При соответствующих предположениях о виде операторов $\widehat{S}$ и $\widehat{U}[15]$ имеют место неравенства Боголюбова

$$
\frac{\lambda}{V_{L}}\langle\widehat{U}\rangle_{\widehat{S}_{\lambda}} \leqslant P_{\beta, \mu}[\widehat{S}]-P_{\beta, \mu}\left[\widehat{S}_{\lambda}\right] \leqslant \frac{\lambda}{V_{L}}\langle\widehat{U}\rangle_{\widehat{S}}
$$

Пусть $\widehat{H}_{\alpha}^{L}=\widehat{H}^{L}-\alpha \widehat{N}$, где гамильтониан $\widehat{H}^{L}$ задан соотношением (1) и параметр $\alpha>0$ будет фиксирован ниже. Соотношение (6) приводит к неравенствам

$$
-\alpha\langle\hat{n}\rangle_{\hat{H}_{\alpha}^{L}}+\frac{a}{r}\left\langle\hat{n}^{r}\right\rangle_{\hat{H}_{\alpha}^{L}} \leqslant P_{\beta, \mu}\left[\widehat{H}_{0}\right]-P_{\beta, \mu}\left[\widehat{H}_{\alpha}^{L}\right] \leqslant-\alpha\langle\hat{n}\rangle_{\hat{H}_{0}}+\frac{a}{r}\left\langle\hat{n}^{r}\right\rangle_{\hat{H}_{0}} .
$$

Рассмотрим эти неравенства в области $\mu<0$. В этом случае $\left\langle\hat{n}^{r}\right\rangle_{\widehat{H}_{0}}=\langle\hat{n}\rangle_{\widehat{H}_{0}}^{r}$. Поскольку для произвольного $r \geqslant 2$ функция $s(x)=x^{r}$ при $x>0$ непрерывно дифференцируема и выпукла вниз, то в силу неравенства Иенсена [17]

$$
\left\langle\hat{n}^{r}\right\rangle_{\widehat{H}_{\alpha}^{L}} \geqslant\langle\hat{n}\rangle_{\widehat{H}_{\alpha}^{L}}^{r}
$$

Тогда неравенства (7) после предельного перехода $L \rightarrow \infty$ примут вид

$$
-\alpha \rho+\frac{a}{r} \rho^{r} \leqslant \lim _{L \rightarrow \infty}\left(P_{\beta, \mu}\left[\widehat{H}_{0}\right]-P_{\beta, \mu}\left[\widehat{H}_{\alpha}^{L}\right]\right) \leqslant-\alpha \rho_{0}+\frac{a}{r} \rho_{0}^{r},
$$


где $\rho_{0}$ обозначает среднюю плотность идеального бозе-газа в бесконечном объеме при $\mu<0$.

Выберем теперь параметр $\alpha$ из условия

$$
\alpha(\rho)=\frac{a}{r}\left(\frac{\rho^{r}-\rho_{0}^{r}}{\rho-\rho_{0}}\right)
$$

Тогда

$$
\lim _{L \rightarrow \infty} P_{\beta, \mu}\left[\widehat{H}_{\alpha}^{L}\right]=\lim _{L \rightarrow \infty} P_{\beta, \mu}\left[\widehat{H}_{0}\right]+\frac{a}{r}\left(\frac{\rho^{r}-\rho_{0}^{r}}{\rho-\rho_{0}}\right) \rho_{0}-\frac{a}{r} \rho_{0}^{r}
$$

Рассмотрим предел $\rho \rightarrow \rho_{0}$, при этом

$$
\lim _{\rho \rightarrow \rho_{0}} \alpha(\rho)=a \rho_{0}^{r-1} \equiv \tilde{\alpha}
$$

и

$$
\lim _{L \rightarrow \infty} P_{\beta, \mu}\left[\widehat{H}_{\tilde{\alpha}}^{L}\right]=\lim _{L \rightarrow \infty} P_{\beta, \mu}\left[\widehat{H}_{0}\right]+a \frac{r-1}{r} \rho_{0}^{r} .
$$

Кроме того, очевидно, что

$$
\lim _{L \rightarrow \infty} P_{\beta, \mu}\left[\widehat{H}_{\tilde{\alpha}}^{L}\right]=\lim _{L \rightarrow \infty} P_{\beta, \mu^{\prime}}\left[\widehat{H}^{L}\right]
$$

где $\mu^{\prime}=\mu+\tilde{\alpha}$.

Взяв гамильтониан

$$
\widehat{H}_{\alpha}^{L}\left(\left\{\frac{h_{j}}{\sqrt{V_{L}}}\right\}\right)
$$

вместо гамильтониана $\widehat{H}_{\alpha}^{L}$ в соотношении (8), получаем

$$
\begin{gathered}
\lim _{L \rightarrow \infty} P_{\beta, \mu^{\prime}}\left[\widehat{H}^{L}\left(\left\{\frac{h_{j}}{\sqrt{V_{L}}}\right\}\right)\right]=\lim _{L \rightarrow \infty} P_{\beta, \mu}\left[\widehat{H}_{0}\left(\left\{\frac{h_{j}}{\sqrt{V_{L}}}\right\}\right)\right]+a \frac{r-1}{r} \rho_{0}^{r} \leqslant \\
\leqslant \lim _{L \rightarrow \infty} P_{\beta, \mu}\left[\widehat{H}_{0}\right]+a \frac{r-1}{r} \rho_{0}^{r}=\lim _{L \rightarrow \infty} P_{\beta, \mu^{\prime}}\left[\widehat{H}^{L}\right] .
\end{gathered}
$$

Таким образом, неравенства Боголюбова (6) позволяют при $\mu<0$ получить соотношение

$$
\lim _{L \rightarrow \infty} P_{\beta, \tilde{\alpha}+\mu}\left[\widehat{H}^{L}\left(\left\{\frac{h_{j}}{\sqrt{V_{L}}}\right\}\right)\right] \leqslant \lim _{L \rightarrow \infty} P_{\beta, \tilde{\alpha}+\mu}\left[\widehat{H}^{L}\right]
$$

являющееся следствием условия гауссовой доминантности, доказанного выше при произвольных $\mu$. 


\section{4. БОЗЕ-КОНДЕНСАЦИЯ В МОДЕЛИ ХУАНГА-ДЭВИСА}

В работах $[12,13]$ изучалась модель неидеального бозе-газа Хуанга-Дэвиса в импульсном представлении вторичного квантования, гамильтониан которой имеет вид

$$
\widehat{H}=\sum_{p}(\lambda(p)-\mu) \hat{a}_{p}^{\dagger} \hat{a}_{p}+\widehat{H}_{1},
$$

где $\lambda(p)$ - неотрицательная функция, $p \in R^{\nu}$ и

$$
\widehat{H}_{1}=\frac{a}{2 V_{L}} \widehat{N}^{2}, \quad \widehat{N}=\sum_{p} \hat{a}_{p}^{\dagger} \hat{a}_{p} \equiv \sum_{p} \hat{n}_{p}
$$

Макроскопическое заполнение основного состояния (бозе-конденсация) имеет место в данной системе при выполнении условия

$$
\Delta \equiv \lim _{L \rightarrow \infty} \frac{1}{V_{L}}\left\langle\hat{a}_{0}^{\dagger} \hat{a}_{0}\right\rangle_{\hat{H}} \neq 0 .
$$

В этом разделе мы, используя неравенство Репсторфа [18], получим точное значение критической температуры в модели (9), тем самым уточнив результаты работы [12].

В работе [12] при условии $\mu \leqslant a \rho$ была найдена верхняя оценка для внутреннего произведения Боголюбова

$$
\left(\hat{a}_{p}^{\dagger}, \hat{a}_{p}\right) \leqslant \frac{1}{\beta(\lambda(p)+a \rho-\mu)},
$$

где по определению

$$
(\hat{A}, \widehat{B})=Z^{-1} \int_{0}^{1} \operatorname{Tr}\left[\widehat{A} e^{-\beta(1-x) \widehat{H}} \widehat{B} e^{-\beta x \widehat{H}}\right]
$$

для произвольных операторов $\hat{A}$ и $\widehat{B}$. Оценка (11) является непосредственным следствием тождества

$$
1=\left\langle\left[\hat{a}_{p}, \hat{a}_{p}^{\dagger}\right]\right\rangle=\left(\hat{a}_{p},\left[\beta \widehat{H}, \hat{a}_{p}^{\dagger}\right]\right)
$$

и неравенства

$$
\left(\hat{a}_{p}^{\dagger}, \hat{n} \hat{a}_{p}\right) \geqslant\langle\hat{n}\rangle\left(\hat{a}_{p}^{\dagger}, \hat{a}_{p}\right) .
$$

Заметим, что неравенство (11) сильнее неравенства

$$
\left(\hat{a}_{p}^{\dagger}, \hat{a}_{p}\right) \leqslant \frac{1}{\beta \lambda(p)},
$$

следуюшего из условия гауссовой доминантности (5).

Используя стандартную процедуру, описанную в работе [12], можно получить следующее неравенство для температурного среднего:

$$
\left\langle\hat{a}_{p}^{\dagger} \hat{a}_{p}\right\rangle_{\hat{H}^{L}} \leqslant \frac{1}{e^{\beta(\lambda(p)+a \rho-\mu)}-1},
$$


где $p \neq 0, \mu \leqslant a \rho$.

Воспользуемся теперь неравенством Репсторфа, являюшимся уточнением известного неравенства Боголюбова,

$$
\left\langle\hat{A}^{\dagger} \hat{A}\right\rangle \geqslant\left\langle\left[\hat{A}, \hat{A}^{\dagger}\right]\right\rangle\left[\exp \left(\frac{\beta\left\langle\left[\hat{A}^{\dagger},[\widehat{H}, \hat{A}]\right]\right\rangle\left\langle\left[\hat{A}, \hat{A}^{\dagger}\right]\right\rangle}{\left|\left\langle\left[\hat{A}, \hat{A}^{\dagger}\right]\right\rangle\right|^{2}}\right)-1\right]^{-1}
$$

Выбрав $\hat{A}=\hat{a}_{p}$ в неравенстве (13), находим

$$
\left\langle\hat{a}_{p}^{\dagger} \hat{a}_{p}\right\rangle_{\hat{H}^{L}} \geqslant \frac{1}{e^{\beta(\lambda(p)+a \rho-\mu)}-1},
$$

где $p \neq 0, \mu \leqslant a \rho$. Сравнивая соотношения (12) и (14), видим, что в термодинамическом пределе нами получено точное значение среднего $\left\langle\hat{a}_{p}^{\dagger} \hat{a}_{p}\right\rangle$.

Теорема 2 [12]. В модели Хуанга-Дәвиса условие (10) выполняется при $\nu \geqslant 3$ и $\beta>\beta_{\mathrm{c}}$, где $\beta_{\mathrm{c}}$ есть единственное решение уравнения

$$
\rho_{\mathrm{c}}=\frac{1}{(2 \pi)^{\nu}} \int \frac{d^{\nu} p}{e^{\beta_{\mathrm{c}} \lambda(p)}-1}
$$

$u \mu_{\mathrm{c}}=a \rho_{\mathrm{c}}$.

Поскольку выше значение парного коррелятора $\left\langle\hat{a}_{p}^{\dagger} \hat{a}_{p}\right\rangle$ было найдено точно, можно утверж дать, что величина $\beta_{\mathrm{c}}$ в уравнении (15) дает точное значение критической температуры модели. Таким образом, при $\mu_{\mathrm{c}}=a \rho_{\mathrm{c}}$ и $\rho>\rho_{\mathrm{c}}$ имеем для плотности конденсата

$$
\Delta= \begin{cases}0, & \text { если } \beta \leqslant \beta_{\mathrm{c}}, \\ \rho-\rho_{\mathrm{c}}, & \text { если } \beta>\beta_{\mathrm{c}} .\end{cases}
$$

При $\lambda(p)=p^{2} / 2$ и $\nu=3$ уравнение (15) принимает вид

$$
\rho_{\mathrm{c}}=\frac{\zeta\left(\frac{3}{2}\right)}{\left(2 \pi \beta_{\mathrm{c}}\right)^{\frac{3}{2}}},
$$

где дзета-функция Римана

$$
\zeta(z)=\sum_{n=1}^{\infty} n^{-z}, \quad \operatorname{Re} z>1 .
$$

Данньй результат совпадает с соответствуюшим точным результатом работы [2]. 


\section{5. МЕТОД БОЛЬШИХ УКЛОНЕНИЙ В ОБОБШЕННОЙ МОДЕЛИ ХУАНГА-ДЭВИСА}

Применим метод больших уклонений $[7,19]$ для изучения введенной в разделе 2 обобшенной модели Хуанга-Дэвиса. Этот метод был использован ранее при рассмотрении стандартной модели Хуанга-Дэвиса $[9,10]$.

Гамильтониан рассматриваемой модели неидеального бозе-газа диагонален по операторам чисел заполнения $\hat{n}_{j}=\hat{a}_{j}^{\dagger} \hat{a}_{j}$. Поэтому можно рассматривать эти числа как случайные переменные, а не как операторы. В этом и состоит основная идея применения методов теории вероятностей при изучении данного типа моделей.

Следуя работе [10], рассмотрим вероятностное пространство $\Omega$, элементами которого $\omega \in \Omega$ являются последовательности $\{\omega(j) \in N \mid j=1,2, \ldots\}$ целых неотришательных чисел, удовлетворяюшие условию

$$
\sum_{j \geqslant 1} \omega(j)<\infty
$$

Основными случайными величинами в развиваемом подходе являются числа заполнения $\left\{\sigma_{j} \mid j=1,2, \ldots\right\}$, задаваемые отображением $\sigma_{j}: \Omega \rightarrow N$, которое определено как $\sigma_{j}(\omega)=\omega(j)$ для каждого $\omega \in \Omega$. Полное число частиц в конфигурации $\omega$ есть

$$
N(\omega)=\sum_{j \geqslant 1} \sigma_{j}(\omega)
$$

Гамильтониан идеального бозе-газа в этих обозначениях для каждого $L \geqslant 1$ принимает вид

$$
H_{L}^{0}(\omega)=\sum_{j \geqslant 1} \lambda_{L}(j) \sigma_{j}(\omega)
$$

где $0=\lambda_{L}(1)<\lambda_{L}(2) \leqslant \cdots$ и $\lambda_{L}(j)=E_{L}(j)-E_{L}(1)$. Система, как и ранее, заключена в области $\Lambda_{L}$ конечного объема $V_{L}$.

Обобшенная полиномиальная модель Хуанга-Дэвиса задается гамильтонианом

$$
\widetilde{H}_{L}(\omega)=H_{L}^{0}(\omega)+a \frac{N_{L}^{r}(\omega)}{r V_{L}^{r-1}},
$$

где $r \geqslant 1, N_{L}(\omega)=\sum_{j} \sigma_{j}(\omega)$. В работах $[9,10]$ был рассмотрен случай $r=2$.

Введем плотность числа частиц $X_{L}=N_{L} / V_{L}$ и функцию

$$
u_{\alpha}(x)=(\mu-\alpha) x-\frac{a x^{r}}{r} .
$$

Так же, как и в статье [10], получаем

$$
\tilde{p}_{L}(\mu)=p_{L}(\alpha)+\frac{1}{\beta V_{L}} \ln \int_{[0, \infty)} \exp \left(\beta V_{L}\left(u_{\alpha} \circ X_{\Lambda}\right)(x)\right) K_{L}^{\alpha}(d x)
$$


для любого фиксированного $\alpha<0$ и для любого действительного $\mu$. Здесь $K_{L}^{\alpha}=P_{L}^{\alpha}$ 。 $X_{L}^{-1}$ есть вероятностная мера на полуоси $[0, \infty)$, а $P_{L}^{\alpha}[\cdot]-$ мера идеального бозе-газа в большом каноническом ансамбле,

$$
P_{L}^{\alpha}[\omega]=\exp \left\{\beta\left[\alpha N(\omega)-H_{L}^{0}(\omega)-V_{L} p_{L}(\alpha)\right]\right\} .
$$

Функция $p_{L}(\mu)$ - давление идеального бозе-газа,

$$
p_{L}(\mu)=\left(\beta V_{L}\right)^{-1} \sum_{j \geqslant 1} \ln \left\{1-\exp \left[\beta\left(\mu-\lambda_{L}(j)\right)\right]\right\}^{-1}, \quad \mu<0 .
$$

Заметим, что отображение $x \rightarrow u_{\alpha}(x)$ непрерывно и ограничено сверху на полуоси $[0, \infty)$ и что множество мер $\left\{K_{L}^{\alpha}, L=1,2, \ldots\right\}$ удовлетворяет принципу больших уклонений с весовой функцией

$$
I^{\alpha}(x)=p(\alpha)+f(x)-\alpha x,
$$

где

$$
f(x)=\sup _{b}\{b x-p(b)\}
$$

есть преобразование Лежандра функции $p(\mu)$, а

$$
p(\alpha)=\lim _{L \rightarrow \infty} p_{L}(\alpha)
$$

(см. теорему 1 в работе [11]). Следовательно, по первой теореме Варадана предел

$$
\tilde{p}(\mu)=\lim _{L \rightarrow \infty} \tilde{p}_{L}(\mu)
$$

существует при следующих предположениях:

ПРЕДПОЛОЖЕНИЕ 1. Предел $\phi(\beta)=\lim _{L \rightarrow \infty} \phi_{L}(\beta)$ cyществует при всех $\beta \in$ $(0, \infty)$, әде функиия распределения $F_{L}(\lambda)=\left(V_{L}\right)^{-1} \max \left\{j: \lambda_{L}(j) \leqslant \lambda\right\} u \phi_{L}(\beta)=$ $\int_{[0, \infty)} \exp (-\beta \lambda) d F_{L}(\lambda)$.

ПрЕДПОЛОЖЕНИЕ 2. Предел $\phi(\beta)$ отличен от нуля хотя бъ для одного значения $\beta \in(0, \infty)$.

ТЕОРема 3. Пусть выполнены предположения 1 и 2. Тогда для конечной критической плотности $\rho_{\mathrm{c}}$ имеют место равенства

$$
\begin{gathered}
\tilde{p}(\mu)=a \frac{r-1}{r}\left(p^{\prime}\left(a_{0}\right)\right)^{r}+p\left(a_{0}\right), \\
\lim _{\delta \searrow 0} \lim _{L \rightarrow \infty} \widetilde{E}_{L}^{\mu}\left[X_{L}(\delta)\right]=\left(\rho-\rho_{\mathrm{c}}\right)^{+},
\end{gathered}
$$

где $\widetilde{E}_{L}^{\mu}[\cdot]$ - математическое ожсидание по отношению к большому каноническому ансамблю с гамильтонианом $\widetilde{H}_{L}$ обобщенной плотности

$$
X_{L}(\delta)=\left(V_{L}\right)^{-1} \sum_{\left\{j: \lambda_{L}(j) \leqslant \delta\right\}} \sigma_{j}(\omega),
$$

$\rho=(\tilde{p})^{\prime}(\mu)$ и точка $a_{0}=a_{0}(\mu)$ определена соотношением $a\left[p^{\prime}\left(a_{0}\right)\right]^{r-1}=\mu-a_{0}$. 
ДокАЗАТЕЛЬСтво. Из соотношения (16) по теореме Варадана имеем для предельного давления

$$
\begin{aligned}
\tilde{p}(\mu) & =p(\alpha)+\sup _{x}\left\{u_{\alpha}(x)-I^{\alpha}(x)\right\}= \\
& =p(\alpha)+\sup _{x}\left\{(\mu-\alpha) x-a \frac{x^{r}}{r}-p(\alpha)-f(x)+\alpha x\right\}= \\
& =\sup _{x}\left\{\mu x-f(x)-a \frac{x^{r}}{r}\right\}=\sup _{x}\{\mu x-\tilde{f}(x)\},
\end{aligned}
$$

где $\tilde{f}(x)=f(x)+a x^{r} / r$.

Можно записать, что

$$
\begin{aligned}
\sup _{x}\left\{\mu x-\sup _{b}\{b x-p(b)\}-a \frac{x^{r}}{r}\right\} & =\sup _{x}\left\{\mu x-a_{0} x+p\left(a_{0}\right)-a \frac{x^{r}}{r}\right\}= \\
& =\sup _{x}\left\{\left(\mu-a_{0}\right) x-a \frac{x^{r}}{r}\right\}+p\left(a_{0}\right),
\end{aligned}
$$

где $a_{0} x-p\left(a_{0}\right)=\sup _{b}\{b x-p(b)\}$. Точка экстремума в соотношении (20) определяется равенством

$$
x_{0}=\left(\frac{\mu-a_{0}}{a}\right)^{\frac{1}{r-1}} .
$$

Следовательно, имеем

$$
\tilde{p}(\mu)=a\left(\frac{\mu-a_{0}}{a}\right)^{1+\frac{1}{r-1}}-\frac{a}{r}\left(\frac{\mu-a_{0}}{a}\right)^{\frac{r}{r-1}}+p\left(a_{0}\right)=a \frac{r-1}{r}\left(\frac{\mu-a_{0}}{a}\right)^{\frac{r}{r-1}}+p\left(a_{0}\right) .
$$

Однако точка $a_{0}$ такова, что $x_{0}=p^{\prime}\left(a_{0}\right)$, т.е. $p^{\prime}\left(a_{0}\right)=\left(\left(\mu-a_{0}\right) / a\right)^{1 /(r-1)}$. Поэтому давление в нашей модели следуюшим образом выражается через давление идеального бозе-газа (ср. эту формулу с формулой (8), полученной ранее другим методом):

$$
\tilde{p}(\mu)=a \frac{r-1}{r}\left(p^{\prime}\left(a_{0}\right)\right)^{r}+p\left(a_{0}\right) .
$$

Таким образом, соотношение (17) доказано.

Докажем теперь наличие обобшенной конденсации (18). Запишем выражение для обобщенной плотности в виде

$$
V_{L} X_{L}(\delta)=V_{L} X_{L}-\sum_{\left\{j: \lambda_{L}(j)>\delta\right\}} \sigma_{j}(\omega)
$$

Тогда

$$
\widetilde{E}_{L}^{\mu}\left[\exp \left\{\beta s V_{L} X_{L}(\delta)\right\}\right]=\exp \left\{\beta V_{L}\left[\tilde{p}_{L}(\mu ; s, \delta)-\tilde{p}_{L}(\mu)\right]\right\}
$$


где $\tilde{p}_{L}(\mu ; s, \delta)$ - давление, соответствующее гамильтониану

$$
\widetilde{H}_{L}(s, \delta)=\widetilde{H}_{L}-s V_{L} X_{L}(\delta)
$$

Из соотношения (21) следует, что

$$
\lim _{L \rightarrow \infty} \widetilde{E}_{L}^{\mu}\left[X_{L}(\delta)\right]=\left.\frac{\partial \tilde{p}(\mu ; s, \delta)}{\partial s}\right|_{s=0}
$$

Воспользуемся формулой (17) и соотношением

$$
p(\mu ; s, \delta)=\int_{[0, \delta]} p(\mu \mid \lambda) d F(\lambda)+\int_{[\delta, \infty)} p(\mu \mid \lambda+s) d F(\lambda)
$$

где $p(\mu \mid s)$ - условное давление идеального газа, определенное равенством

$$
\beta p(\mu \mid \lambda)=-\ln [1-\exp (\beta(\mu-\lambda))]
$$

Получим, что

$$
\left.\frac{\partial \tilde{p}(\mu ; s, \delta)}{\partial s}\right|_{s=0}=\left(\frac{\mu-a_{0}}{a}\right)^{\frac{1}{r-1}}-\int_{[\delta, \infty)} p^{\prime}\left(a_{0} \mid \lambda\right) d F(\lambda)
$$

где точка $a_{0}$ определена в формулировке теоремы 3. Из формулы (22) следует требуемое утверждение, т.к.

$$
\left.\lim _{\delta \rightarrow 0} \frac{\partial \tilde{p}(\mu ; s, \delta)}{\partial s}\right|_{s=0}= \begin{cases}0, & \text { если } \mu<a \rho_{\mathrm{c}}^{r-1} \\ \rho-\rho_{\mathrm{c}}, & \text { если } \mu \geqslant a \rho_{\mathrm{c}}^{r-1}\end{cases}
$$

где

$$
\rho_{\mathrm{c}}=\int_{[0, \infty)} p^{\prime}(0 \mid \lambda) d F(\lambda)
$$

совпадает с критической плотностью идеального бозе-газа. Теорема 3 доказана.

Анализ полученных результатов позволяет установить, что давление в исследуемой модели при данной плотности является возрастаюшей функцией как параметра $r$, так и константы взаимодействия $a$. Количество же конденсата не зависит ни от $a$, ни от $r$ и совпадает с количеством конденсата идеального бозе-газа при той же плотности.

Благодарности. Данная работа частично финансировалась кафедрой математики (Стохастический анализ и математическая физика, Чили), фондом Fapesp 1997 /11508-0 (Бразилия) и РФФИ, проект 99-01-00887 (Россия). 


\section{Список литературы}

[1] К. Хуанг. Статистическая механика. М.: Мир, 1966.

[2] E. B. Davies. Commun. Math. Phys. 1972. V. 28. P. 69.

[3] A. С. Швари. Тр. Моск. мат. общ-ва. 1970. Т. 22. С. 165.

[4] J. L. Lebowitz, O. Penrose. J. Math. Phys. 1966. V. 7. P. 98.

[5] L. J. Landau, I. E. Wilde. Commun. Math. Phys. 1970. V. 70. P. 43.

[6] M. van den Berg, J. T. Lewis, J. V. Pulé. Helv. Phys. Acta. 1986. V. 59. P. 1271.

[7] S. R. S. Varadhan. Commun. Pure Appl. Math. 1966. V. 19. P. 261.

[8] M. van den Berg, J. T. Lewis, J. V. Pulé. Commun. Math. Phys. 1988. V. 116. P. 1.

[9] M. van den Berg, J. T. Lewis. Limit Theorems for Stochastic Processes associated with a Boson Gas. In: Lect. Notes in Math. V. 1325. Stochastic Mechanics and Stochastic Processes. Proc. Conf., Swansea/UK, 1986. Eds. A. Truman, I. M. Davies. Berlin: Springer, 1988. P. 16.

[10] M. van den Berg, J. T. Lewis, P. J. Smedt. J. Stat. Phys. 1984. V. 37. P. 697.

[11] M. van den Berg, J. T. Lewis, J. V. Pulé. Large Deviations and the Boson Gas. In: Lect. Notes in Math. V. 1325. Stochastic Mechanics and Stochastic Processes. Proc. Conf., Swansea/UK, 1986. Eds. A. Truman, I. M. Davies. Berlin: Springer, 1988. P. 24.

[12] М. Корджини ${ }^{1)}$, Д. П. Санкович. ТМФ. 1996. Т. 108. С. 421.

[13] M. Corgini, D. P. Sankovich. Int. J. Mod. Phys. B. 1997. V. 11. P. 3329.

[14] Ф. А. Березин. Метод вторичного квантования. М.: Наука, 1986.

[15] Н. Н. Боголюбов (мл.), Й. Г. Бранков, В. А. Загребнов, А. М. Курбатов, Н. С. Тончев. Метод аппроксимирующего гамильтониана в статистической физике. София: Изд-во БАН, 1981.

[16] М. Рид, Б. Саймон. Методы современной математической физики. Т. 2. М.: Мир, 1978.

[17] Г. Харди, Джс. Литтлвуд, Г. Полиа. Неравенства. М.: ГИИЛ, 1948.

[18] G. Roepstorff. Commun. Math. Phys. 1977. V. 53. P. 143.

[19] R. Ellis. Entropy, large deviations and statistical mechanics. Berlin, Heidelberg, New York: Springer, 1985.

Поступила в редакцию 10. XII.1998 г., после доработки 3.II.1999 г.

\footnotetext{
1) M. Corgini
} 